\title{
Age concerns
}

DOI:

10.1038/nrn2203

\section{URL:}

Entrez Gene: http:// eutils.ncbi.nlm.nih. gov/entrez/query. fcgi?db=gene

\section{SIRT2:}

http://www.ncbi. nlm.nih.gov/sites/ entrez?Db=gene\&C $\mathrm{md}=$ ShowDetailVie w\&TermToSearch= 22933Eordinalpos= 38itool=EntrezSyst em2.PEntrez.Gene. Gene_ResultsPanel. Gene_RVDocSum
It is well known that aging increases the risk of developing a neurodegenerative disease. As our knowledge of the molecular basis of aging grows, common pathways for aging and neurodegeneration may be found. Two papers published this month provide links between aging-related changes in neurons and the development of Parkinson's disease (PD), pointing to approaches to protect or rescue vulnerable neuronal populations.

One group of proteins that might be involved in aging and neurodegeneration are the sirtuins, a family of histone deacetylases that regulate aging in many organisms. Kazantsev and colleagues provide evidence for a link between sirtuins and the development of $\alpha$-synuclein-containing inclusions in the substantia nigra pars compacta ( $\mathrm{SNc}$ ), which is associated with PD pathology. The authors identified compounds that specifically inhibit sirtuin 2 (SIRT2) and that protected human neuroglioma (H4) cells and primary dopaminergic cell cultures from $\alpha$-synuclein-mediated toxicity. Furthermore, treatment with small interfering RNA against SIRT2 replicated these effects in $\mathrm{H} 4$ cells. The authors tested two structurally diverse SIRT2 inhibitors, AGK2 and AK-1, in a Drosophila model of PD in which $\alpha$-synuclein is overexpressed in the brain, and observed the rescue of dopaminergic neurons. This protective effect involves the formation of larger $\alpha$-synuclein inclusions, which is thought to minimize the interactions of $\alpha$-synuclein with cellular proteins, in comparison with those of multiple smaller inclusions.
Although it is unknown how SIRT2 affects $\alpha$-synuclein aggregation, these findings suggest that SIRT2 might be a useful target for PD.

Surmeier and colleagues asked why SNc dopaminergic neurons are particularly vulnerable to neurodegeneration. Working from the observation that juvenile SNc neurons are less vulnerable in animal models of PD, they investigated the changes that occur when these neurons age. $\mathrm{SNc}$ neurons have some distinctive characteristics, including an autonomous pacemaking activity that maintains basal levels of dopamine release in regions that are innervated by these neurons, such as the striatum. The authors demonstrated an age-related shift in the type of ion channel involved in this pacemaking activity in mice: juvenile SNc neurons relied on sodium and HCN (hyperpolarisation-activated and cyclic-nucleotide-gated cation) channels, whereas adult $\mathrm{SNc}$ neurons required $\mathrm{Ca}_{\mathrm{v}} 1.3$ channels of the L-type voltage-dependent calcium channel family.

Reasoning that the associated increase in calcium influx might contribute to the vulnerability of aging SNc neurons to toxic insults, Surmeier and colleagues investigated the effects of blocking $\mathrm{Ca}_{\mathrm{v}} 1.3$ channel activity using isradipine in brain slices from adult mice. After several hours of isradipine-induced inactivity, $\mathrm{SNc}$ neurons regained pacemaking activity, but were now reliant on sodium and HCN channel activity. This 'rejuvenation' was associated with increased neuroprotection of dopaminergic neurons against

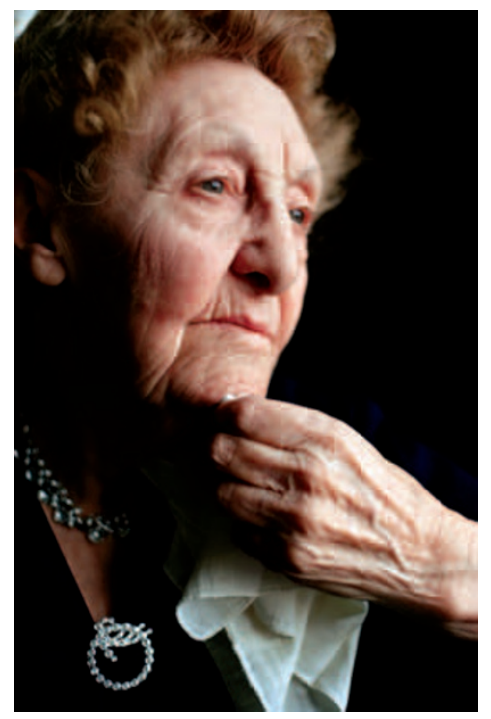

the mitochondrial toxin rotenone. Furthermore, the authors were able to repeat the rejuvenation in vivo, and they observed neuroprotection against acute 6-hydroxydopamine (6-OHDA) striatal injections and chronic MPTP treatment. As isradipine is already used clinically for the treatment of hypertension and stroke, clinical testing for its use against PD could rapidly be initiated.

As the average age of the population continues to rise, the need to develop therapeutic or preventive strategies for neurodegenerative diseases becomes more urgent. These two papers show that insights into the age-related changes that occur in the brain and the molecular processes by which these changes are regulated can provide promising avenues for such research.

Katherine Whalley

ORIGINAL RESEARCH PAPERS Savio Chan, C. et al. 'Rejuvenation' protects neurons in mouse models of Parkinson's disease. Nature 447, 10811086 (2007) | Fleming Outeiro, T. et al. Sirtuin 2 inhibitors rescue $\alpha$-synuclein-mediated toxicity in models of Parkinson's disease. Science 21 June 2007 (doi:10.1126/science.1143780) 